Journal of

Synchrotron

Radiation

ISSN 0909-0495

Received 5 July 2010

Accepted 20 August 2010

\section{Single-crystal Raman spectroscopy and X-ray crystallography at beamline X26-C of the NSLS}

\author{
Deborah Stoner-Ma,* John M. Skinner, Dieter K. Schneider, Matt Cowan, \\ Robert M. Sweet and Allen M. Orville*
}

Biology Department, Brookhaven National Laboratory, Upton, NY 11973, USA. E-mail: dstonerma@bnl.gov, amorv@bnl.gov

\begin{abstract}
Three-dimensional structures derived from X-ray diffraction of protein crystals provide a wealth of information. Features and interactions important for the function of macromolecules can be deduced and catalytic mechanisms postulated. Still, many questions can remain, for example regarding metal oxidation states and the interpretation of 'mystery density', i.e. ambiguous or unknown features within the electron density maps, especially at $\sim 2 \AA$ resolutions typical of most macromolecular structures. Beamline X26-C at the National Synchrotron Light Source (NSLS), Brookhaven National Laboratory (BNL), provides researchers with the opportunity to not only determine the atomic structure of their samples but also to explore the electronic and vibrational characteristics of the sample before, during and after X-ray diffraction data collection. When samples are maintained under cryo-conditions, an opportunity to promote and follow photochemical reactions in situ as a function of X-ray exposure is also provided. Plans are in place to further expand the capabilities at beamline X26-C and to develop beamlines at NSLS-II, currently under construction at BNL, which will provide users access to a wide array of complementary spectroscopic methods in addition to high-quality X-ray diffraction data.
\end{abstract}

Keywords: Raman; single-crystal spectroscopy; X-ray diffraction.

\section{Introduction}

Understanding the complex relationships among atomic structure, electronic structure and chemistry is crucial for obtaining insights into the fundamental biological processes including those that $(a)$ underlie human diseases and $(b)$ can contribute to the development of clean energy sources. A variety of spectroscopic methods can provide a means with which to explore these relationships, particularly when the sample includes a conjugated cofactor or ligand. Indeed, approximately one-third of all macromolecules expressed in organisms contain a non-protein cofactor (Waldron et al., 2009). Cofactors with color, such as metal ions and/or organic molecules, are present in approximately 11000 entries in the Protein Data Bank archive as of June 2010. These macromolecules usually exhibit a characteristic optical absorption spectrum that often changes during catalysis and/or during $\mathrm{X}$-ray diffraction data collection. Post-translational modifications can also result in unique spectroscopic characteristics. Spectroscopic studies are also useful for proteins co-crystallized with substrate or product analogs (De la Mora-Rey \& Wilmot, 2007).

To conduct concurrent studies utilizing both crystallographic and spectroscopic methods, an integrated infra- structure is required at the $\mathrm{X}$-ray beamline. Such a facility has been developed at beamline X26-C of the National Synchrotron Light Source (NSLS) and other synchrotrons (Owen et al., 2009; McGeehan et al., 2009; Davies et al., 2009; Ellis et al., 2008; Royant et al., 2007; Carpentier et al., 2007). Beamline $\mathrm{X} 26-\mathrm{C}$ is currently capable of following changes in the visible absorption spectrum and in the vibrational spectrum via Raman spectroscopy as a function of X-ray exposure. The design and use of the recently added Raman system is presented herein; a separate article will present the design and implementation of correlated absorption spectroscopy with X-ray diffraction (Orville et al., 2011).

\section{Materials and methods}

\subsection{Instrumentation}

The facility at beamline X26-C at NSLS supports correlated $\mathrm{X}$-ray diffraction and spectroscopic analysis of macromolecules, utilizing specifically visible absorption spectroscopy and Raman vibrational spectroscopy, each at a $90^{\circ}$ orientation to the X-ray beam. The instrumentation and methodology for single-crystal visible absorption spectroscopy 
has already been described (Héroux et al., 2009; Orville et al., 2009, 2011; Yi et al., 2010).

In this report we focus on Raman spectroscopy. We currently collect offresonance Raman spectra utilizing a frequency-stabilized multimode $785 \mathrm{~nm}$ diode laser (Process Instruments, Salt Lake City, UT, USA) and near-resonance Raman spectra with a diodepumped solid-state single longitudinalmode $532 \mathrm{~nm}$ laser (Laser Quantum, Cheshire, UK). Laser line widths are $30 \mathrm{GHz}$ (multimode) and $1 \mathrm{MHz}$, respectively. The beam is delivered via a $100 \mu \mathrm{m}$-diameter fiber optic to a wavelength-specific Horiba Jobin-Yvon (Edison, NJ, USA) Raman SuperHead. The SuperHead contains an edge filter to both select the excitation wavelength and to remove that wavelength from the collected scattered signal. An Olympus SLMPlanN $50 \times / 0.35$ objective is used to focus the beam to a $\sim 25 \mu \mathrm{m}$ spot size. The focal depth, $f$, is $3.6 \mu \mathrm{m}$. The region of the crystal probed is the same as that used for both X-ray diffraction and absorption data collection, as will be discussed below. The Raman SuperHead and the collection objective for absorbance mode are located below the crystal on an $x, y$ and $z$ translation stage (Fig. 1). This motorized stage allows for rapid switching between alignments optimized for electronic absorption and for Raman spectroscopy. Reproducibility of the stage position was determined by the manufacturer (CrystalLogic, CA, USA) to be within $2.5 \mu \mathrm{m}$ in each of $x, y$ and $z$. The SuperHead collects both Rayleigh and Raman scattered light in a backscatter $\left(180^{\circ}\right)$ mode. An edge filter within the SuperHead removes the Rayleigh scatter. The Raman scattered light is focused into another $100 \mu \mathrm{m}$ fiber optic which connects to an iHR320 spectrometer (Horiba Jobin-Yvon). The entrance slit width is adjustable with a maximal width of $2 \mathrm{~mm}$. Three gratings on a motorized turret are available: 600, 1200 and 1800 lines $\mathrm{mm}^{-1}$. The user can easily switch between the collection of broad low-resolution spectra and the collection of high-resolution spectra from a narrow region of particular interest. The detector is a Peltiercooled (203 K) Synapse CCD detector (Horiba Jobin-Yvon). The CCD chip is a front-illuminated open electrode $1024 \times$ 256 pixel array with a $26 \times 26 \mu \mathrm{m}$ pixel size. Laser calibration is assured using a Ne calibration lamp (Oriel, Stratford, CT, USA) or fluorescent room light. Overall instrument resolution using the 1200 grating is $4 \mathrm{~cm}^{-1}$ (Horiba specifications).

Of vital importance to the success of correlated studies is the co-localization of the crystal rotation axis, the X-ray beam and the focal point of each spectroscopic method such that an identical region of the crystal is probed. Two cameras are used for alignment. One camera views the crystal from $35^{\circ}$ below the X-ray path and is used for centering the crystal to the
$\mathrm{X}$-ray beam. This camera is also used to view the alignment of the focal points of the spectroscopy objectives on a 400/10 micromesh (MiteGen, Ithaca, NY, USA). A second line-ofsight camera is contained within each of the Raman SuperHeads and facilitates aligning the Raman lasers to the crystal eucentric rotation point and the X-ray beam. Exposure of the mesh to the laser must be minimized to prevent melting of the mesh; typical exposure times are brief $(<10 \mathrm{~s})$ with the laser attenuated to deliver $1 \mathrm{~mW}$.

\subsection{Software integration}

Software control for absorption spectroscopy has been fully incorporated into the beamline control software (Yi et al., 2010; Orville et al., 2011). Raman data acquisition is currently controlled by LabSpec 5.55.10, a Windows-based software provided by Horiba Jobin-Yvon. Future plans include transferring control of data acquisition to the beamline control software in a manner similar to that done for absorption spectroscopy.

\subsection{Data collection}

After the crystal is centered for X-ray diffraction collection, the crystal orientation providing the optimal Raman spectrum is typically determined manually by collecting $10 \mathrm{~s}$ spectra at approximately $10^{\circ}$ increments of rotation, with the laser attenuated such that only the strongest Raman vibration modes are observed. This is to avoid laser-induced damage to the crystal. Crystal orientations that result in the lowest cleanest background and the best signal-to-noise ratio are identified. Power levels and exposure times also have to be optimized for each sample, as tolerance to the laser will vary 


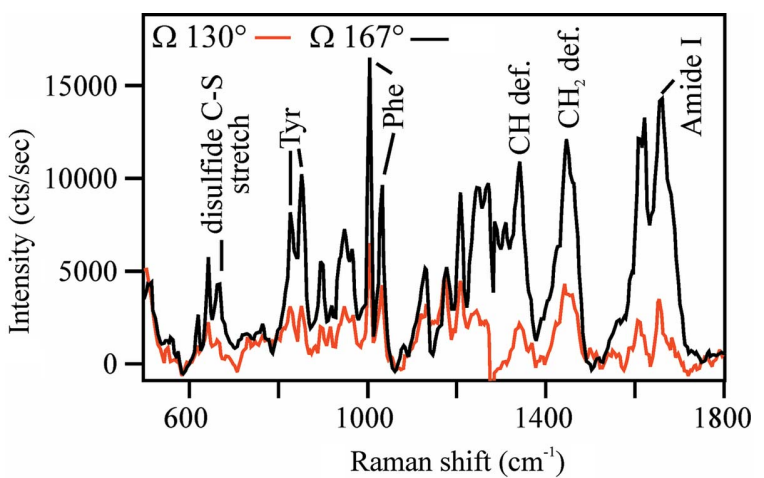

Figure 2

Raman spectra of a $\mathrm{Zn}^{2+}$ insulin crystal. Raman spectra were taken at $\Omega=$ $130^{\circ}$ (red) and $167^{\circ}$ (black) where $\Omega=0^{\circ}$ is defined as one of the angles at which the flat face of the crystal is orthogonal to the Raman objective. The spectra were collected using $6 \mathrm{~mW}$ of $532 \mathrm{~nm}$ laser excitation and $50 \mathrm{~s}$ total acquisition time. Peaks assignments are based on Yu et al. (1972).

from sample to sample. Typical conditions for a full protein Raman spectrum acquisition include total collection times of 1 to $5 \mathrm{~min}$ and laser powers of $2-10 \mathrm{~mW}$ at the sample.

\section{Results and discussion}

\subsection{Preliminary Raman experiments}

3.1.1. $\mathbf{Z n}^{2+}$ insulin. Use of the Raman system at X26-C was inaugurated during the April 2010 RapiData crystallography course run by the Macromolecular Crystallography Research Resource (PXRR) at BNL. Raman spectra using the $532 \mathrm{~nm}$ laser source were collected on several protein crystals, including a $\mathrm{Zn}^{2+}$ insulin crystal provided by the course (Soares et al., 2003). The importance of investigating the crystal orientation angle $(\Omega)$ relative to the X-ray beam axis was immediately recognized from the insulin spectra taken at the two angles, $130^{\circ}$ and $167^{\circ}$ (Fig. 2). Both the intensity and quality of the spectra varied greatly. We and others have suggested that anisotropic absorption and the prism effects of the single crystal impact electronic absorption and Raman scattering (Carey, 2006; Pearson et al., 2007). If a planar chromophore is present within the crystal, that too impacts the intensity of Raman scattering of individual modes (Stoner-Ma et al., 2006). It is also important to reduce the likelihood of artefacts inherent to the sample mount; for example, the nylon loop should not intersect the Raman laser beam path. To prevent loop contributions, we often collect absorption data with the flat face of the loop perpendicular to the spectroscopy beam. This orientation also frequently presents the broadest face of the crystal to the beam. The optimal orientation for Raman spectroscopy is not necessarily the same as that for absorption spectroscopy. In the case of $\mathrm{Zn}^{2+}$ insulin, the approximate perpendicular orientation was when $\Omega$ was set to $105^{\circ}$. However, a better Raman spectrum was collected at an $\Omega$ angle of $167^{\circ}$, i.e. closer to a parallel than perpendicular orientation of the loop to the laser beam. Comparison of the obtained $\mathrm{Zn}^{2+}$ insulin spectrum with a published Raman spectrum of insulin (Yu et al., 1972) allows assignment of several of the normal modes, as indicated in Fig. 2. The quality of the spectrum obtained from a protein such as insulin, which lacks a chromophore, indicates that X-ray-induced vibrational changes, if they occur, may be discernible by non-resonant Raman spectroscopy. An example might include the breakage of a disulfide bond and concomitant loss of the $\mathrm{S}-\mathrm{S}$ mode at approximately $510 \mathrm{~cm}^{-1}$. Non-resonant Raman spectroscopy has already been used to follow X-ray damage in brominated DNA (McGeehan et al., 2007). Intensity changes of a carbonbromine mode were noted as well as some normal modes shifts of $10-17 \mathrm{~cm}^{-1}$. In protein samples, changes of this magnitude may also be observed. An example would be shifts owing to changes in hydrogen bonding or salt bridges within the protein. Assignment of the normal modes responsible for changes observed in proteins would likely require theoretical calculations, difference Raman spectroscopy and/or isotope editing.

3.1.2. Orientation dependence. The role of sample orientation relative to the laser beam has been examined further using a frozen glass of a buffer and cryo-protectant commonly used in macromolecular crystallography. A loop holding a cryo-cooled thin film (approximately $15 \mu \mathrm{m}$ ) of $50 \%$ glycerol $(v / v)$ and $0.5 M$ sodium cacodylate $\mathrm{pH} 8$ was oriented such that when the loop $\Omega$ angle was set to $0^{\circ}$ a flat side of the loop was orthogonal to the laser beam. The resulting spectra showed great variation in the intensity of the glycerol $\mathrm{C}-\mathrm{O}$ stretch,

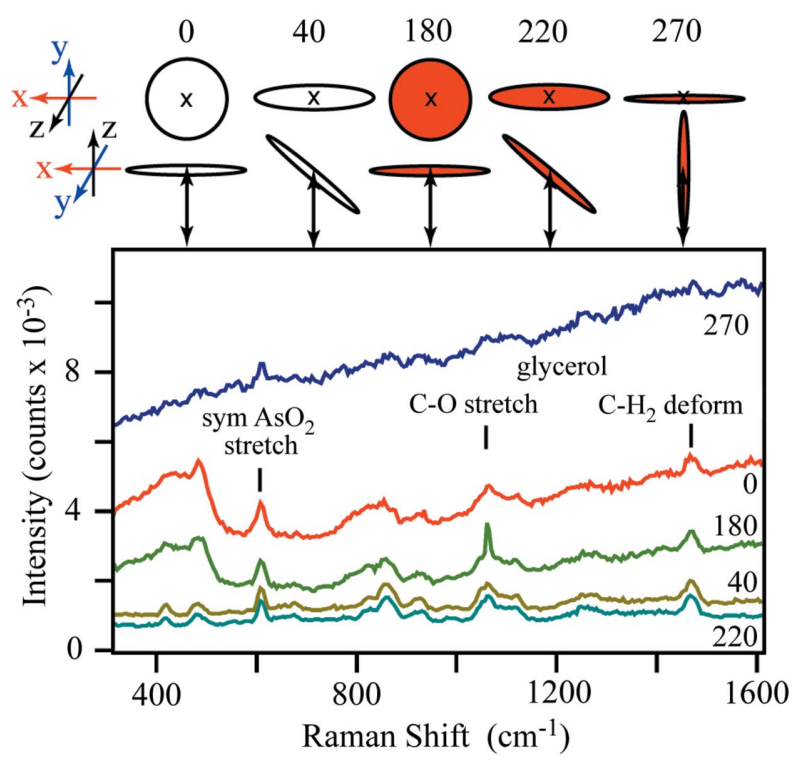

Figure 3

Raman spectra of frozen $50 \%$ glycerol, $0.5 \mathrm{M}$ cacodylate $\mathrm{pH} 8$ using $2.6 \mathrm{~mW} 532 \mathrm{~nm}$ laser excitation and a $10 \mathrm{~s}$ collection time. Spectra were collected every $10^{\circ}$ of loop rotation about the omega axis $(\Omega)$. Selected spectra are shown to illustrate the dependence of spectral quality on $\Omega$ : $0^{\circ}$ (red), $40^{\circ}$ (olive), $180^{\circ}$ (green), $220^{\circ}$ (light blue) and $270^{\circ}$ (dark blue). The top of the figure indicates loop orientation at each $\Omega$, with the red and white color scheme distinguishing the two flat faces. The right-handed coordinate system at beamline $\mathrm{X} 26-\mathrm{C}$ has the $\mathrm{X}$-ray beam travelling along $x$, the crystal rotation axis parallel to $y$, and the Raman spectroscopy axis along $z$. The 600 lines $\mathrm{mm}^{-1}$ grating and a $1 \mathrm{~mm}$ spectrometer entrance slit size were used. The assignment of the symmetric $\mathrm{AsO}_{2}$ stretch of cacodylate is from Thuy et al. (2010); those for glycerol are from Mendelovici et al. (2000). 
glycerol $\mathrm{CH}_{2}$ deformation and $\mathrm{AsO}_{2}$ symmetric stretch modes as $\Omega$ is changed (Fig. 3). While, again, it might be assumed that orienting the flat face of the sample orthogonal to the laser beam (i.e. $0^{\circ}$ and $180^{\circ} \Omega$ ) would yield the best spectra, this is clearly not the case. The $0^{\circ}$ and $180^{\circ} \Omega$ spectra do contain the strong glycerol and cacodylate modes, but they also exhibit a high background signal, especially in the $400-500 \mathrm{~cm}^{-1}$ region. The higher background may be a result of prism effects of the sample. The intense and broad $400-500 \mathrm{~cm}^{-1}$ feature has been observed in spectra from other samples, including protein crystals, and may serve as an indicator of an un-optimized $\Omega$. Both spectra in which the loop is edge-on (i.e. $90^{\circ}$ and $270^{\circ} \Omega$ ) to the laser beam show very high backgrounds with only barely identifiable vibrational modes from the sample. Contributors to these results include an un-optimized focal point and contributions or reflections from the loop itself. The $270^{\circ} \Omega$ spectrum is included in Fig. 3; the $90^{\circ}$ spectrum is not shown due to saturation of the detector. The best spectrum in terms of low background and strong sample peaks was obtained at $\Omega=220^{\circ}$. Rotating the sample $180^{\circ}$ further to $\Omega=$ $40^{\circ}$ also yielded a clean spectrum. For this specimen, therefore, these are the angles of choice for the Raman spectroscopy, and not the $\Omega$ angles of $0^{\circ}$ and $180^{\circ}$ as one might easily assume. Similar results have been observed for several protein crystals in which the optimal angle for Raman collection was not face on (vide infra). With protein crystals, there will be additional contributions to the Raman spectra, including the orientation-dependent sample volume probed and possible fluorescence. Our results indicate that assumptions cannot be made as to the optimal Raman orientation but needs to be determined for each and every sample.

\subsection{Current studies}

Collaborative studies incorporating Raman collection together with absorption and diffraction data are now underway. Results from studies on a Rieske iron-sulfur protein have shown specific vibrational mode shifts following $\mathrm{X}$-ray exposure, clearly indicating a rapid reduction upon $\mathrm{X}$-ray exposure. These results will be published shortly. Similar experiments with a variety of other iron-containing proteins are planned. Studies will be continued on the choline oxidase system in which a FAD $\mathrm{C} 4 \mathrm{a}-\mathrm{OOH}$ or $\mathrm{C} 4 \mathrm{a}-\mathrm{OOH}$ adduct had been identified using absorption spectroscopy and X-ray diffraction structural data (Orville et al., 2009).

Moving forward, we will continue to expand the capabilities at X26-C to include fluorescence (steady-state and timeresolved) spectroscopy and to provide a wider selection of excitation wavelengths for Raman spectroscopy (633 and $442 \mathrm{~nm}$ lasers are currently planned). Controls for Raman acquisition will be fully integrated into the beamline control software, as has been done for absorption spectroscopy.
A complementary off-line spectroscopy facility is under construction immediately adjacent to the beamline. Lastly, with the completion of NSLS-II expected in 2015, a proposal has been submitted for a fully integrated spectroscopyenhanced X-ray beamline. This beamline, if approved and funded, would maximally exploit the potential of this new third-generation $3 \mathrm{GeV}$ synchrotron.

This work was supported by the Office of Biological and Environmental Research, US Department of Energy and the National Center for Research Resources (2 P41 RR012408) of the National Institutes of Health. The authors are grateful for the support of the entire PXRR staff.

\section{References}

Carey, P. R. (2006). Annu. Rev. Phys. Chem. 57, 527-554.

Carpentier, P., Royant, A., Ohana, J. \& Bourgeois, D. (2007). J. Appl. Cryst. 40, 1113-1122.

Davies, R. J., Burghammer, M. \& Riekel, C. (2009). J. Synchrotron Rad. 16, 22-29.

De la Mora-Rey, T. \& Wilmot, C. M. (2007). Curr. Opin. Struct. Biol. 17, 580-586.

Ellis, M. J., Buffey, S. G., Hough, M. A. \& Hasnain, S. S. (2008). J. Synchrotron Rad. 15, 433-439.

Héroux, A., Bozinovski, D. M., Valley, M. P., Fitzpatrick, P. F. \& Orville, A. M. (2009). Biochemistry, 48, 3407-3416.

McGeehan, J. E., Carpentier, P., Royant, A., Bourgeois, D. \& Ravelli, R. B. G. (2007). J. Synchrotron Rad. 14, 99-108.

McGeehan, J., Ravelli, R. B. G., Murray, J. W., Owen, R. L., Cipriani, F., McSweeney, S., Weik, M. \& Garman, E. F. (2009). J. Synchrotron Rad. 16, 163-172.

Mendelovici, E., Frost, R. L. \& Kloprogge, T. (2000). J. Raman Spectrosc. 31, 1121-1126.

Orville, A. M., Lountos, G. T., Finnegan, S., Gadda, G. \& Prabhakar, R. (2009). Biochemistry, 48, 720-728.

Orville, A. M., Stoner-Ma, D., Skinner, J. M., Héroux, A., Schneider, D. K. \& Sweet, R. M. (2011). J. Synchrotron Rad. In preparation.

Owen, R. L., Pearson, A. R., Meents, A., Boehler, P., Thominet, V. \& Schulze-Briese, C. (2009). J. Synchrotron Rad. 16, 173-182.

Pearson, A. R., Pahl, R., Kovaleva, E. G., Davidson, V. L. \& Wilmot, C. M. (2007). J. Synchrotron Rad. 14, 92-98.

Royant, A., Carpentier, P., Ohana, J., McGeehan, J., Paetzold, B., Noirclerc-Savoye, M., Vernède, X., Adam, V. \& Bourgeois, D. (2007). J. Appl. Cryst. 40, 1105-1112.

Soares, A. S., Caspar, D. L. D., Weckert, E., Héroux, A., Hölzer, K., Schroer, K., Zellner, J., Schneider, D., Nolan, W. \& Sweet, R. M. (2003). Acta Cryst. D59, 1716-1724.

Stoner-Ma, D., Melief, E. H., Nappa, J., Ronayne, K. L., Tonge, P. J. \& Meech, S. R. (2006). J. Phys. Chem. B, 110, 22009-22018.

Thuy, N. T. B., Yokogawa, R., Yoshimura, Y., Fujimoto, K., Koyan, M. \& Maenosono, S. (2010). Analyst, 135, 595-602.

Waldron, K. J., Rutherford, J. C., Ford, D. \& Robinson, N. J. (2009). Nature (London), 460, 823-830.

Yi, J., Orville, A. M., Skinner, J., Skinner, M. \& Richter-Addo, G. B. (2010). Biochemistry, 49, 5969-5971.

Yu, N.-T., Liu, C. S., Culver, J. \& O'Shea, D. C. (1972). Biochim. Biophys. Acta, 263, 1-6. 